\title{
51. Experiences of Cases with Tumors lying within and adjacent to Incisura Tentorii
}

\author{
Kenzo Matsuoka, Tohru Uozumi, Ryoichi Nozaki, \\ Shun-nosuke Minami, Muneo Kajimura and Saburo SaKaki \\ The First Department of Surgery, Osaka University \\ Kazuo Hara, Hayao Shiga and Yasusada Fujino \\ Department of Radiology, Osaka University
}

\begin{abstract}
Operations to remove tumors of incisural region must be based on a very accurate preoperative anatomic diagnosis. The lesion must be evaluated as to size, exact location in relation to the brain stem, the edge of tentorium, the middle fossa and the major vessels.

In this report, five cases of this region were presented; that is, hemangioblastoma and astrocytoma of the anterior cerebellum, neurinoma of the fifth nerve, glioma of the midbrain and pinealoma of parasellar portion. Air capping on tumors and shift of third and fourth ventricles on fractional pneumoencephalograms and tumor stain, widening, spraying, dislocation and stretch of posterior cerebral and superior cerebellar arteries on vertebral angiograms were observed.

Temporal approaches must be selected for tumors of the anterior and lateral portions of this region, and occipital approaches for those of the posteaior portion with tentorial incision, if neccessary.
\end{abstract}

\section{Intracranial Invasion of Malignant Neoplasmas}

\author{
Yasuo Koyama \\ National Cancer Center Hospital
}

1. Intracranial malignant lesions were found in 93 to 637 patients with extracranial malignant primary foci, examined on autopsy at National Cancer Center Hospital.

2. Intracranial metastases were founn in 74 cases which consisted of 45 parenchymal metastases, 29 meningeal metastases (23 of which were meningeal metastases alone) and 13 pituitary metastases (6 of which were pituitary metastasis alone). The incidence of intracranial metastasis was 